\title{
Anatomical and Radiological Analyses of L-shaped Kidney with Vascular Anomalies
}

\author{
JOE IWANAGA, KOICHI WATANABE, TSUYOSHI SAGA, NOBUHIRO TAHARA*, \\ YOKO TABIRA, AYANO SAKURAGI, KEIKO KAJI, KIYOSHI TAKAHASHI \\ AND KOH-ICHI YAMAKI \\ Department of Anatomy and *Division of Cardio-Vascular Medicine, Department of Medicine, \\ Kurume University School of Medicine, Kurume, 830-0011 Japan \\ Received 11 February 2016, accepted 7 February 2017 \\ J-STAGE advance publication 12 June 2017 \\ Edited by TSUKASA IGAWA
}

\begin{abstract}
Summary: L-shaped kidney is a congenital anomaly. The disorder results in the kidney appearing very similar in shape to horseshoe kidney (also a congenital anomaly), but either the right or left kidney is located at a position lower than the other kidney. In this report, we describe a woman with L-shaped kidney, identified during anatomical dissection, and compare the findings with clinical data obtained before her death. We discuss the embryology of L-shaped kidney based on detailed anatomical data on the kidney and its vascular system obtained by means of gross anatomical, radiological, and histological examinations. Our findings indicate the importance of detailed anatomical information when planning surgical procedures in patients with fused kidneys, as well as kidney transplantation, resection of renal carcinoma, or surgical treatment of abdominal aortic aneurysm.
\end{abstract}

Key words crossed fused renal ectopia, L-shaped kidney, horseshoe kidney, transplantation

\section{INTRODUCTION}

L-shaped kidney is one form of crossed fused renal ectopia. L-shaped kidney is most commonly found accidentally during imaging or anatomical dissection. In this report, we describe the anatomical and radiological findings of a woman with L-shaped kidney following dissection at our medical school, and show the importance of preoperative anatomical findings in planning surgical procedures in patients with fused kidneys.

\section{CASE REPORT}

A 79-year-old woman with systemic sarcoidosis donated her body to medical research at our department. She died from multiple organ failure resulting from hepatic cell carcinoma. Her liver was hypertrophied and indurated. During an anatomical dissection course for students, it was observed that she had Lshaped kidney. We were able to obtain prior clinical data, such as computed tomography images, from our hospital. Two images were available that had been taken on two previous occasions before she died, one at 2 months before her death and the other at 14 months. The kidney was resected and investigated in detail. The study was performed in accordance with the principles contained in the Declaration of Helsinki (64th WMA General Assembly, Fortaleza, Brazil, October 2013).

\section{Shape, size, and position of the kidney}

The L-shaped kidney after resection is shown in Fig. 1A. In the right kidney, the superior border was

Corresponding Author: Joe Iwanaga, D.D.S., Ph.D., Department of Anatomy, Kurume University School of Medicine, 67 Asahi-machi, Kurume, Fukuoka 830-0011, Japan. Tel: +81-942-31-7540, Fax: +81-942-33-3233, E-mail: iwanaga_jyou@med.kurume-u.ac.jp

Abbreviations: AA, abdominal aorta; BC, Bowman's capsule; CFRE, Crossed fused renal ectopia; CT, coeliac trunk; GC, glomerular capillary; IMA, inferior mesenteric artery; IVC, inferior vena cava; LAG, left adrenal gland; LCIV, left common iliac vein; LEIV, left external iliac vein; LIIV, left internal iliac vein; LOV, left ovarian vein; LRA, left renal artery; LU, left ureter; RAG, right adrenal gland; REIV, right external iliac vein; RIIV, right internal iliac vein; ROV, ovarian vein; RRA, right renal artery; RU, right ureter; SMA, superior mesenteric artery. 
between the third and fourth lumbar vertebrae. In the left kidney, the superior border was at the middle part of the first lumbar vertebra, and the inferior border of the kidney was at the lower part of the fifth lumbar vertebra. The fixed kidney weighed $312.3 \mathrm{~g}$. The thickness of the right kidney was $2.0 \mathrm{~cm}$ and that of

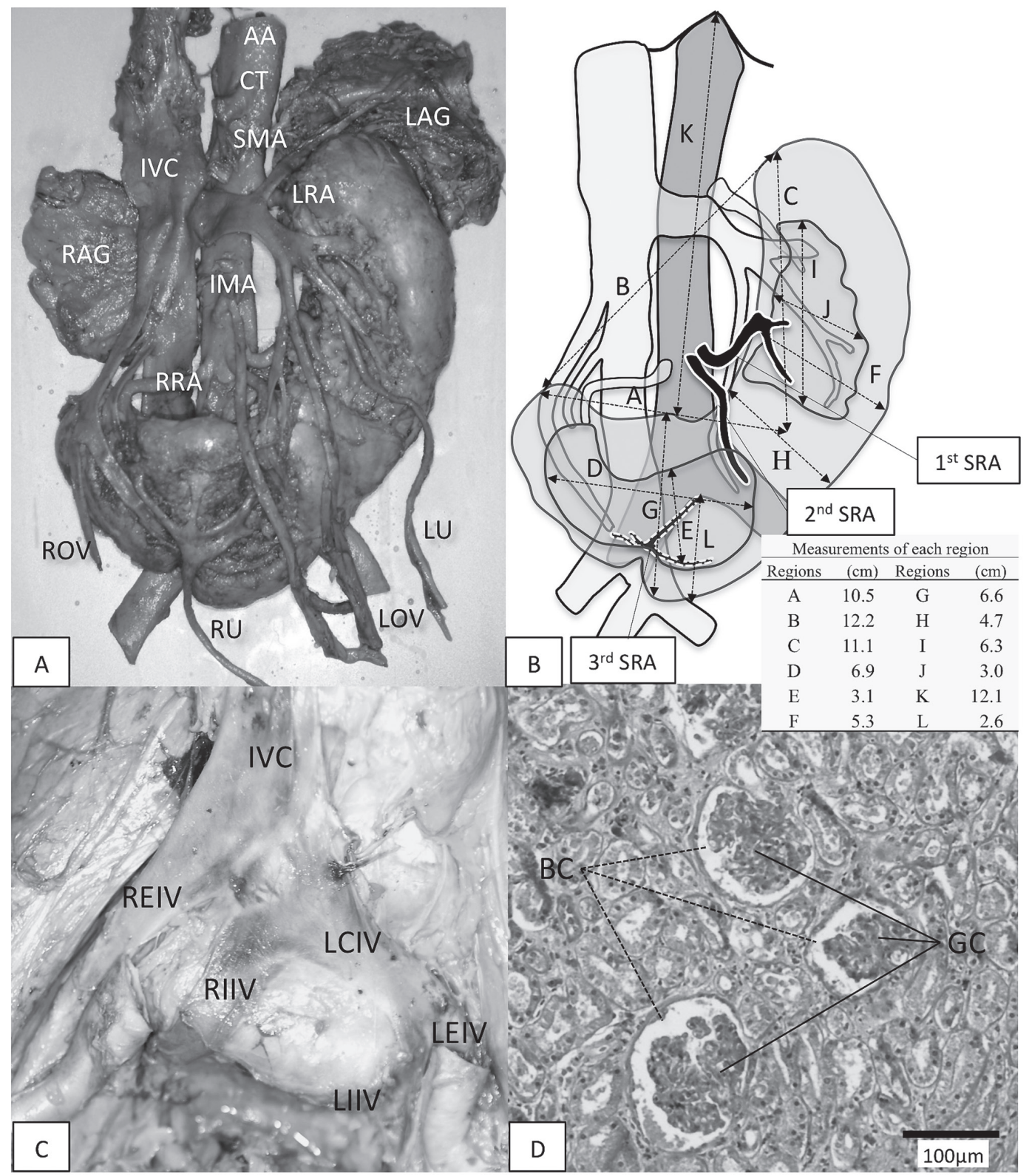

Fig. 1. Gross anatomical and microscopic observations of the L-shaped kidney.

A: L-shaped kidney after removal of lower digestive tract. B: Measurements of each region of the L-shaped kidney. Results are presented in a table at the lower right corner of Fig. 1B. Black vessels signify surplus renal arteries. C: The venous system at the fifth lumbar vertebra. The right common iliac vein is absent and the right internal iliac vein flows directly into the left common iliac vein. D: Histology of the isthmus as seen with light microscopy. Many renal corpuscles and renal tubules are observed. Scale bar $=100 \mu \mathrm{m}$.

AA, abdominal aorta; BC, Bowman's capsule; CT, coeliac trunk; GC, glomerular capillary; IMA, inferior mesenteric artery; IVC, inferior vena cava; LAG, left adrenal gland; LCIV, left common iliac vein; LEIV, left external iliac vein; LIIV, left internal iliac vein; LOV, left ovarian vein; LRA, left renal artery; LU, left ureter; RAG, right adrenal gland; REIV, right external iliac vein; RIIV, right internal iliac vein; ROV, ovarian vein; RRA, right renal artery; $\mathrm{RU}$, right ureter; SMA, superior mesenteric artery. 
the left was $4.1 \mathrm{~cm}$. The isthmus was maximally 2.7 $\mathrm{cm}$ thick. Other measurements are shown in Fig. 1B.

\section{Renal pelvis and ureter}

The right renal pelvis was slightly enlarged, and continued until the right ureter, which descended almost to the midline ventrally from the right kidney without forming any shallow groove on the ventral surface of the kidney (Fig. 1A). The left renal pelvis was normal and continued until the left ureter, which descended ventrally from the left kidney.

\section{Vascular system}

The celiac trunk, superior mesenteric artery (SMA), and inferior mesenteric artery (IMA) branched off from the ventral side of the abdominal aorta (AA) without any anomalies. The right renal artery arose from the right side of the AA and was positioned lower than the IMA, and passed over the ventral to the inferior vena cava (IVC) and continued to the upper part of the right kidney. The left renal artery arose from the left side of the AA at a slightly lower level to that of the SMA, and entered into the upper part of the left renal pelvis (Fig. 1B).

Three surplus renal arteries (SRA) entered the kidney (Fig. 1B). The first SRA (5.3 mm in diameter) arose from the ventral side of the AA, at a position slightly higher than that of the right renal artery, and divided into two branches that entered the lower part of the left renal hilum. The second SRA ( $4.3 \mathrm{~mm}$ in diameter) arose from the ventral side of the AA, at the same level of the first SRA, and descended ventrally to the kidney, before entering the inferior part of the right renal hilum. The third SRA (3.6 $\mathrm{mm}$ in diameter) arose from the bifurcation point of the common iliac arteries, descended dorsally to the kidney, and made a deep groove at the inferior border of the kidney within the renal parenchymal tissue, before entering the right renal hilum. There were no abnormalities in either the lumbar arteries or the median sacral artery.

The right internal iliac vein and left common iliac veins joined and ascended, before joining the right external iliac vein at the middle part of the fifth lumbar vertebra behind the right common iliac artery. The right common iliac vein was absent (Fig. 1C).

The IVC ascended dorsally to the isthmus and right side of the AA. The right renal vein joined the right ovarian vein and passed ventrally to the AA, before draining into the IVC.

One surplus renal vein (SRV) arose from the upper part of the right renal hilum and drained into the IVC at the upper part of the third lumbar vertebra just below the right RV.

The left ovarian vein and left adrenal vein drained, respectively, into the lower and upper walls of the left renal vein. The left IVC entered the lateral wall of the IVC at a higher position than the right renal vein, which was at the lower border of the first lumbar vertebra. One of the branches of the left renal vein, which ascended past the medial border of the left kidney, arose from the lower part of the right renal hilum (Fig. 1B).

\section{Histology of the isthmus}

Hematoxylin and eosin staining was performed using paraffin-embedded preparations resected from renal parenchymal tissue from the dorsal side of the

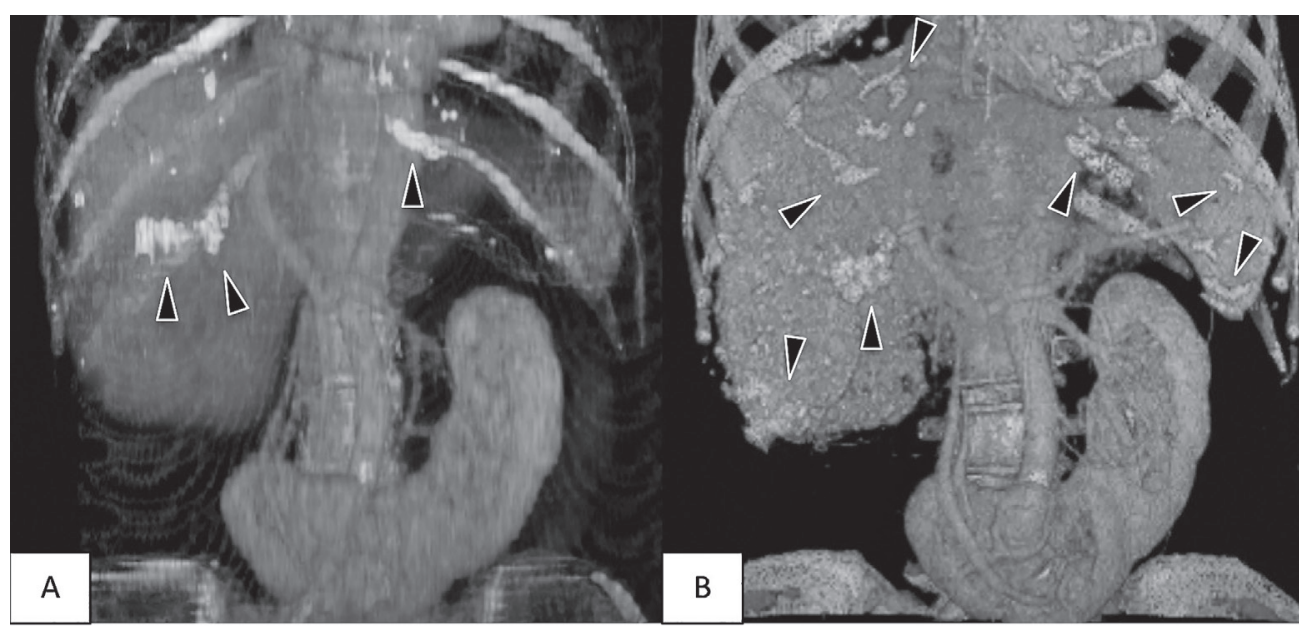

Fig. 2. CT images taken on two occasions prior to the patient's death.

A: CT image of the patient's abdomen 14 months before she died. B: CT image of the patient's abdomen 2 months before she died. Black arrowheads show calcification. 
isthmus. Results from this study showed that the isthmus of this kidney was made from parenchymal structures, like that of the normal kidney (Fig. 1D).

\section{Computed tomography findings}

Two computed tomography images of the kidney taken approximately 14 months and 2 months before the woman died were analyzed. There appeared to be no changes in the position and morphology of the kidney, except the degree of calcification of the liver, between these pictures (Fig. 2A and B). The venous systems, especially the arterial systems, were nearly the same as the dissection findings.

\section{DISCUSSION}

Crossed fused renal ectopia (CFRE) is a relatively uncommon congenital anomaly of the kidney. Autopsy incidence has been calculated at 1 in 2000 [2]. The key morphological feature is transposition of the ectopic kidney to the opposite side, with fusion of the two kidneys [1]. L-shaped kidney is one form of CFRE [7]. It occurs more frequently in men than in women. Crossover of the left kidney to the right side is the most common form of crossed fused renal ectopia [3]. Some have described its detailed anatomy using computed tomography $[7,8]$.

When we observed the L-shaped kidney in the present case, our initial thoughts were that it might be a horseshoe kidney compressed by the hypertrophied liver. However, examination of the two computed tomography images obtained on two occasions prior to the woman's death suggested that changes in the liver had not affected the position and morphology of the kidney. The only change observed was calcification resulting from the progress of hepatic cell carcinoma. This indicated that the L-shaped kidney was probably congenital. Findings of the vascular system, such as the low position of the right renal artery and the left renal vein coming from the right renal hilum, led us to conclude that L-shaped kidney in this case was probably a developmental anomaly. During embryo development, the IVC and the common iliac veins are formed following iliac anastomosis between the 6th and 10th weeks of gestation [9]. The ectopic kidney, which was incapable of ascending, might have disturbed the formation of the right common iliac vein in the present case.

In recent years, numerous abdominal surgeries for patients with fused kidneys have been reported, such as kidney transplantation $[5,10]$, resection of renal carcinoma [6], and surgical treatment of abdominal aortic aneurysm [4]. Almost all cases made mention of the complicated vascular system and isthmus in the surgical procedures. Therefore, preoperative diagnosis based on anatomical findings is very important for surgical intervention in patients with fused kidneys, as well as kidney transplantation, resection of renal carcinoma, or surgical treatment of abdominal aortic aneurysm.

ACKNOWLEDGMENTS: The authors wish to thank those individuals who donated their bodies and tissues for the advancement of education and research.

CONFLICT OF INTEREST: The authors have no conflict of interest to declare.

\section{REFERENCES}

1. Abeshouse BS and Bhisitkul I. Crossed renal ectopia with and without fusion. Urol Int 1959; 9:63-91.

2. Baggenstoss AH. Congenital anomalies of the kidney. Med Clin North Am. 1951; 1:987-1004.

3. Boyan N, Kubat H, and Uzum A. Crossed renal ectopia with fusion: report of two patients. Clin Anat. 2007; 20:699-702.

4. Canova G, Masini R, Santoro E, Bartolomeo S, Martini C et al. Surgical treatment of abdominal aortic aneurysm in association with horseshoe kidney. Three case reports and a review of technique. Tex Heart Inst J. 1998; 25:206-210.

5. Iwanaga J, Saga T, Tabira Y, Watanabe K, and Yamaki KI. Contrast imaging study of the horseshoe kidney for transplantation. Surg Radiol Anat. 2015; 37:1267-1271.

6. Kumar S, Singh S, Jain S, Bora GS, and Singh SK. Robotassisted heminephrectomy for chromophobe renal cell carcinoma in L-shaped fused crossed ectopia: Surgical challenge. Korean J Urol. 2015; 56:729-732.

7. Miclaus GD, Pupca G, Gabriel A, Matusz P, and Loukas M. Right lump kidney with varied vasculature and urinary system revealed by multidetector computed tomographic (MDCT) angiography. Surg Radiol Anat. 2015; 37:859-865.

8. Pupca G, MICLĂUŞ GD, BUCURAŞ V, Iacob N, Sas I et al. Left crossed fused renal ectopia L-shaped kidney type, with double nutcracker syndrome (anterior and posterior). Rom J Morphol Embryol. 2014; 55 (3 Suppl):1237-1241.

9. Sato A. Venous Anomalies and Horseshoe Kidney. Circu J. 2011; 75:2759-2760.

10. Stroosma OB, Smits JM, Schurink GWH, de Boer J, Persijn GG et al. Horseshoe kidney transplantation within the eurotransplant region: a case control study. Transplantation. 2001; 72:1930-1933. 